\title{
VALVE REPAIR FOR MITRAL REGURGITATION ASSOCIATED WITH ISOLATED DOUBLE-ORIFICE MITRAL VALVE
}

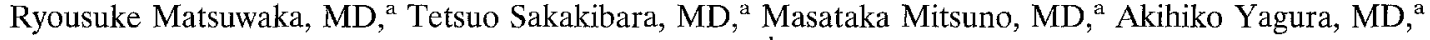 \\ Masato Yoshikawa, MD, ${ }^{\mathrm{a}}$ and Fuminobu Ishikura, MD, ${ }^{\mathrm{b}}$ Osaka, Japan
}

Double-orifice mitral valve (DOMV) can occur as an isolated lesion or in association with other cardiac malformations of which atrioventricular canal defect is most common. ${ }^{1}$ Isolated DOMV is, however, rarely identified when the valve is functioning normally. We present here a case with mitral regurgitation in isolated DOMV in which valve repait was done successfully.

A 55-year-old man was referred to our hospital with exertional dyspnea. Physical examination showed a grade $4 / 6$ holosystolic murmur at the left fourth intercostal space. The electrocardiogram showed atrial fibrillation. The chest $x$-ray film showed a dilated left atrium. Transthoracic two-dimensional and color Doppler echocardiography showed a DOMV with grade 4 mitral regurgitation because of a torn chorda at the mitral orifice of the anterolateral side. The left ventricular diastolic and systolic dimensions by echocardiography were $74 \mathrm{~mm}$ and 42 $\mathrm{mm}$, respectively. Cardiac catheterization revealed a peak pulmonary capillary wedge pressure of $21 \mathrm{~mm} \mathrm{Hg}$. The left ventriculogram showed severe mitral regurgitation.

An operation was done with the use of standard cardiopulmonary bypass with moderate hypothermia $\left(28^{\circ} \mathrm{C}\right)$ and cold cardioplegic arrest. The left atrium was entered through the standard longitudinal incision. The mitral valve was found to have two orifices, which were completely separated by a fibromuscular bridging tissue (Fig. 1). The orifices were almost equal in size: the posteromedial orifice was $30 \mathrm{~mm}$; the anterolateral orifice, $25 \mathrm{~mm}$. Each orifice had its own site of chordal insertion. No cleft was identified on either the anterior or posterior leaflet of each orifice. One half of the posterior leaflet of the anterolateral orifice was found to have prolapse because of a torn chorda. A quadrangular segmental resection of the prolapsed posterior leaflet was done. Large, pledgeted 2-0 sutures were placed at the level of the anulus for plication. The split leaflet was sutured with interrupted 4-0 polypropylene sutures. Because the fibromuscular bridging tissue seemed to be unsuitable for placement of the annuloplasty ring, another pledgeted suture was added to reinforce the plicated anulus. By this procedure,

From the Divisions of Cardiovascular Surgery ${ }^{\mathrm{a}}$ and Cardiology, ${ }^{\mathrm{b}}$ Cardiovascular Center, Osaka Police Hospital, Osaka, Japan.

Received for publication April 22, 1996; accepted for publication May 8, 1996.

J Thorac Cardiovasc Surg 1996;112:1666-7

Copyright (C) 1996 by Mosby-Year Book, Inc.

$0022-5223 / 96 \$ 5.00+0 \quad \mathbf{1 2 / 5 4 / 7 4 9 0 6}$ the anterolateral orifice was reduced to $10 \mathrm{~mm}$ in diameter. Transesophageal Doppler echocardiography showed trivial mitral regurgitation from the repaired anterolateral orifice. The patient was discharged from the hospital on postoperative day 15 after an uneventful recovery.

Various classifications for DOMV have been proposed on the basis of the size and location of the two orifices. ${ }^{1-3}$ The case presented here is an example of a complete type in the classification of Trowitzsch and colleagues ${ }^{2}$ and of a central type in the classification of Cascos, Rabago, and Sokolowski. ${ }^{3}$ In the majority of isolated cases of DOMV, the second orifice is found as an accessory orifice, which is the cause of mitral regurgitation. ${ }^{1,4}$ For this type of accessory orifice, simple closure has been the choice to correct mitral regurgitation. ${ }^{4} \mathrm{Mitral}$ regurgitation associated with isolated DOMV with two equal orifices as in our case has rarely been described in previous literature. ${ }^{5}$ Because no cleft was identified at operation, the choices of treatment seemed to be repair or closure of the valve. In a case with two equal orifices, however, closure of one orifice might produce acute diminution of the mitral valve area leading to a stenotic condition. ${ }^{6}$ In addition, closure of the orifice might possibly cause deformity at the fibrous bridging tissue, leading to incompetence of another intact orifice. Considering the possible problem in closure of the orifice and the finding of a torn chorda located only at the posterior leaflet, valve repair was chosen in our case. The

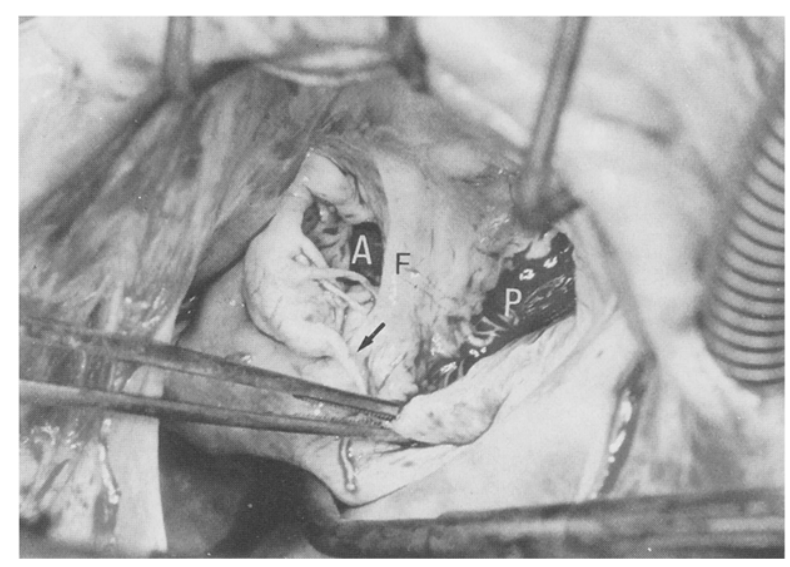

Fig. 1. Intraoperative view of DOMV. $A$, Anterolateral orifice; $P$, posteromedial orifice; $F$, fibromuscular bridging tissue dividing two orifices; arrow, torn chorda at posterior leaflet of anterolateral orifice. 
placement of the annuloplasty ring may be controversial. We prefer to preserve the architecture of the intact anulus without placing the ring at the bridging tissue. To the best of our knowledge, a case with valve repair in isolated DOMV with two equal orifices has not been reported previously. Though the follow-up is still short term in our case, we conclude that valve repair is the choice of treatment for mitral regurgitation in isolated DOMV.

REFERENCES

1. Bano-Rodrigo A, Van Praagh S, Trowitzsch E, Van Praagh R. Double-orifice mitral valve: a study of 27 cases with develop mental, diagnostic and surgical considerations. Am J Cardiol 1988;61:152-60.
2. Trowitzsch E, Bano-Rodrigo A, Burger B, Colan SD, Sanders SP. Two-dimensional echocardiographic findings in double orifice mitral valve. J Am Coll Cardiol 1985;6:383-7.

3. Cascos AS, Rabago P, Sokolowski M. Case report: duplication of the tricuspid valve. Br Heart J 1967;29:943-5.

4. Kenaan G, Neufeld HN, Deutsch V, Shem-Tov A. Isolated congenital double-orifice mitral valve. Isr J Med Sci 1974;10: 743-6.

5. Kawahira M, Sanada J, Kataoka H, Nakamura K, Hashimoto S. A case of double orifice mitral valve complicated by ruptured chordae tendineae. Proc Jap Soc Ulc Med 1992;41: 311-2.

6. Amano J, Suzuki A. Surgical treatment of duplication of the mitral valve. J Cardiovasc Surg 1986;27:323-7.

\title{
PERCUTANEOUS TRANSVENOUS ANGIOPLASTY OF A STENOSED BICAVAL ANASTOMOSIS AFTER ORTHOTOPIC CARDIAC TRANSPLANTATION
}

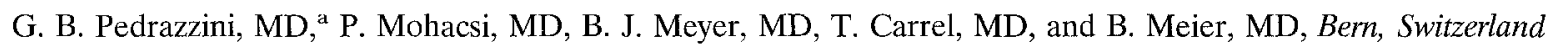

Orthotopic cardiac transplantation is the treatment of choice for patients with end-stage heart failure. The operative technique used at most transplantation centers was developed in the early 1960 s by Lower and Shumway ${ }^{1}$ with a canine model. This original technique includes two atrial anastomoses and anastomosis of the aorta and pulmonary artery. Main advantages of this classical procedure are its simplicity, short operative time, and low prevalence of perioperative and postoperative technical complications.

Mild to moderate regurgitation of the atrioventricular valves (mainly of the tricuspid valve and to a lesser degree of the mitral valve), however, is a common postoperative clinical and echocardiographic finding. These morphologic and functional changes of the normal donor heart are thought to occur because of a postoperative modification in the atrial geometry. Moreover, the technique of biatrial anastomoses seems to increase the occurrence of sinus node dysfunction. ${ }^{2}$

To prevent these potential complications, an alternative technique with bicaval anastomoses rather than the right atrial suture was recently reintroduced by several groups

From the Department of Cardiology ${ }^{\mathrm{a}}$ and Clinic for Thoracic and Cardiovascular Surgery, ${ }^{\mathrm{b}}$ University Hospital, Bern, Switzerland.

Paul Mohacsi supported by a grant from the Swiss National Research Foundation (grant No. 31-39695.1).

Received for publication Feb. 13, 1996; accepted for publication March 25, 1996.

J Thorac Cardiovasc Surg 1996;112:1667-9

Copyright 1996 by Mosby-Year Book, Inc.

$0022-5223 / 96 \$ 5.00+0 \quad 12 / 54 / 73791$ performing for transplantation. This technical refinement was originally described by Webb and colleagues ${ }^{3}$ in 1959 . The anatomic geometry and the sinus node function seem to be better preserved, but this technique can lead to significant stenosis of the venous anastomoses even when an interrupted suture technique is used. This report discusses the case of a patient with a severe stenosis of the superior vena cava (SVC) 2 weeks after heart transplantation. The condition was treated successfully with percutaneous balloon angioplasty.

Cardiac transplantation was performed in a 52-year-old white man because of end-stage dilated cardiomyopathy. Instead of biatrial anastomoses, separate SVC and inferior vena caval anastomoses were performed with interrupted 4.0 polypropylene sutures. A caliber mismatch between the donor and recipient SVCs was noted, with the recipient's SVC three times as large as the donor's SVC. After operation, immediate sinus rhythm was present and no significant atrioventricular regurgitation was demonstrated.

The postoperative course was uneventful, with the exception of cellular rejection (ISHLT $3 \mathrm{~A}$ ) on postoperative day 14 , which was successfully treated with corticosteroids. An endomyocardial biopsy sample was difficult to obtain, however, because it was nearly impossible to introduce a guide wire through the SVC into the right atrium. After several attempts, successful introduction of a $6 \mathrm{~F}$ catheter revealed a pressure gradient of $10 \mathrm{~mm} \mathrm{Hg}$ across the stenosis (Fig. 1). The tight stenosis was confirmed by SVC angiography, and it was decided to use percutaneous angioplasty to dilate the anastomosis at the SVC level.

For this purpose, a $9 \mathrm{~F}$ sheath and a Trefoil $3 \times 6 \mathrm{~mm}$ balloon (Schneider AG, Bulach, Switzerland) was used. 